\title{
Synthesis of PdNi Bimetallic Nanoparticles and Their Composites in Reverse Micelles
}

\author{
M. O. Sergeev ${ }^{a, *}$, A. A. Revina ${ }^{a, b}$, and O. V. Suvorova ${ }^{a}$ \\ ${ }^{a}$ Frumkin Institute of Physical Chemistry and Electrochemistry, Russian Academy of Sciences, Moscow, 119071 Russia \\ ${ }^{b}$ Mendeleev University of Chemical Technology of Russia, Moscow, 125047 Russia \\ *e-mail: mihail-ol-sergeev@yandex.ru \\ Received October 12, 2020; revised December 22, 2020; accepted January 12, 2021
}

\begin{abstract}
In this work, results of the synthesis of bimetallic nanoparticles (NPs) based on transition metals $\mathrm{Pd}, \mathrm{Ni}$, and Fe reduced by quercetin in reverse-micellar solution (RMS) of $\mathrm{H}_{2} \mathrm{O} / \mathrm{AOT} /$ isooctane are presented. Using fluorescence spectroscopy, the formation of bimetallic structure of $\mathrm{Pd}_{\text {core }} \mathrm{Ni}_{\text {shell }} \mathrm{NP}$ is shown and a synergetic enhancement of optical absorbance is revealed in the plasmon resonance spectra at the wavelength values intrinsic for monometallic NPs. Investigation of the adsorption of bimetallic NPs on the surface of $\gamma$-alumina proves the advantage of palladium-containing particles with $\mathrm{Fe}_{\text {core }} / \mathrm{Pd}_{\text {shell }}$ obtained as a result of targeted reduction of palladium in RMS on the surface of large Fe NPs, which appears in the increase in the adsorption effectiveness of such a structure due to stronger binding with the carrier surface. This approach decreases the amount of expensive metal during production of palladium nanocomposites through a significant decrease in production costs.
\end{abstract}

Keywords: reverse micelles, palladium, nickel, iron, bimetallic nanoparticles, quercetin

DOI: $10.1134 /$ S2070205121030217

\section{INTRODUCTION}

Various methods for synthesis of bimetallic nanoparticles have been developed over the last decade, and their characteristics have been studied. Bimetallic NPs are widely used in photochemical, electrochemical, and catalytic processes [1, 2]. Improving the characteristics of photochemical processes is related to the presence of localized surface plasmons in NPs and their effect on optical and optical and electrooptical characteristics of other substances, which allows one to use them in photoelectrochemical cells. Increasing the operational characteristics of sensor devices is based on specific electrochemical properties and energy characteristics of NPs. The use of bimetallic NPs reduces the consumption of expensive metal in catalysts, increases their operational stability, and enhances catalytic activity, which is caused by a synergetic effect [3].

In this work, $\mathrm{Ni}$ was chosen as the main metal due to its ability to cleave $\mathrm{C}-\mathrm{C}$ and $\mathrm{C}-\mathrm{H}$ bonds, while $\mathrm{Pd}$ metal was chosen as a synergetic additive due to its important role in catalytic processes with hydrogen and ability to decrease the deactivation of main metal. The effectiveness of the methods for synthesis of corresponding nanocomposites is also an important issue, and an increase in the effectiveness would decrease the cost of the recovery of expensive metals from waste solutions and their further processing.
The aim of this work is to develop effective synthesis of bimetallic PdNi NPs and their nanocomposites, which are perspective for use in fuel cells of ethanol conversion [4], as well as dehydrogenation of hydrocarbons. Possibility of the use of large Fe NPs as carrier particles for palladium NPs in the form of $\mathrm{Fe}_{\text {core }} / \mathrm{Pd}_{\text {shell }}$ during adsorption synthesis of nanocomposites was also considered. This choice is due to the low cost of metal and ease of etching of iron after deposition if necessary.

\section{EXPERIMENTAL}

\section{Materials}

Salts of nickel $\mathrm{NiCl}_{2} \cdot 6 \mathrm{H}_{2} \mathrm{O}$, (analytical grade, $98 \mathrm{wt} \%$, Khimreaktiv), palladium $\mathrm{PdCl}_{2}$ (99.9 wt \%, Aldrich), and iron $\mathrm{FeSO}_{4} \cdot\left(\mathrm{NH}_{4}\right)_{2} \mathrm{SO} \cdot 6 \mathrm{H}_{2} \mathrm{O}$ (chemically pure); quercetin dihydrate $\mathrm{C}_{15} \mathrm{H}_{10} \mathrm{O}_{7} \cdot 2 \mathrm{H}_{2} \mathrm{O}$ (Qr) (98 wt \%, Merck); sodium bis(2-ethylhexyl)sulfosuccinate $\mathrm{C}_{20} \mathrm{H}_{37} \mathrm{NaO}_{7} \mathrm{~S}$ (AOT) (96 wt \%, Acros organics); isooctane (99.95 wt \%, Etalonnyi-1, Komponentreaktiv); $n$-heptane (chemically pure); distilled water; and $\gamma-\mathrm{Al}_{2} \mathrm{O}_{3}$ (powder) as a base for nanocomposites were used in the work. All reagents were used without further purification. 
Table 1. Content of metal ions in reverse-micellar systems

\begin{tabular}{|c|c|c|c|c|c|c|c|c|c|c|}
\hline \multirow{2}{*}{ Specimen } & \multirow[t]{2}{*}{$\omega$} & \multicolumn{3}{|c|}{$\begin{array}{l}\text { Concentration of metal } \\
\text { in water pool, } \mathrm{mol} \mathrm{dm}^{-3}\end{array}$} & \multicolumn{3}{|c|}{$\begin{array}{l}\text { Concentration of metal } \\
\text { in RMS, } \mu \mathrm{mol} \mathrm{dm}{ }^{-3}\end{array}$} & \multicolumn{3}{|c|}{$\begin{array}{l}\text { Concentration of metal ions } \\
\text { in RMS, } \mathrm{mg} \mathrm{dm}^{-3}\end{array}$} \\
\hline & & $\mathrm{Pd}^{2+}$ & $\mathrm{Fe}^{2+}$ & $\mathrm{Ni}^{2+}$ & $\mathrm{Pd}^{2+}$ & $\mathrm{Fe}^{2+}$ & $\mathrm{Ni}^{2+}$ & $\mathrm{Pd}^{2+}$ & $\mathrm{Fe}^{2+}$ & $\mathrm{Ni}^{2+}$ \\
\hline $\mathrm{Pd} N P$ in $\mathrm{RMS}$ & 5 & 0.015 & - & - & 202 & - & - & 21.55 & - & - \\
\hline Ni NP in RMS & 5 & - & - & 0.015 & - & - & 202 & - & - & 11.89 \\
\hline FeNP in RMS & 5 & - & 0.300 & - & - & 4050 & - & - & 226.2 & - \\
\hline PdNi NP in RMS & 5 & 0.0075 & - & 0.0075 & 101 & - & 101 & 10.78 & - & 5.94 \\
\hline $\mathrm{Fe} / \mathrm{Pd} \mathrm{NP}$ in $\mathrm{RMS}$ & 8.7 & 0.0064 & 0.172 & - & 150 & 4010 & - & 15.80 & 224.0 & - \\
\hline
\end{tabular}

\section{Chemical Synthesis of Metal Nanoparticles in Reverse Micelles}

Metal NPs [5] were synthesized according to the procedure from [6] in a basic reverse-micellar solution of $0.15 \mathrm{~mol} \mathrm{dm}^{-3}$ AOT in isooctane in the presence of $150 \mu \mathrm{mol} \mathrm{dm}{ }^{-3}$ Qr and molecular oxygen. Solubilization coefficient $w$ for reverse-micellar solutions (RMSs) corresponded to 5.0 and 8.7 in the case of FePd NPs.

$$
\omega=\frac{\left[\mathrm{H}_{2} \mathrm{O}\right]}{[\mathrm{AOT}]} .
$$

Concentrations of salts and molar ratio of metals $\left(M_{1} / M_{2}\right)$ in RMSs are given in Table 1.

The concentration of metal ions in the water pool of micelle $\left(c_{M_{i}}^{\text {w.p. }}\right)$ in the beginning of the synthesis of monometallic nanoparticles corresponded to $0.015 \mathrm{~mol} \mathrm{dm}^{-3}$, while that of bimetallic PdNi corresponded to $0.0075 \mathrm{~mol} \mathrm{dm}^{-3}$ for each metal; in the case of FePd, the concentration of iron is $0.172 \mathrm{~mol} \mathrm{dm}^{-3}$ and that of palladium is $0.0064 \mathrm{~mol} \mathrm{dm}^{-3}$. These concentrations were calculated using the following equation:

$$
c_{M_{i}}^{\text {w.p. }}=\frac{c_{M_{i}} x_{i} V_{i}}{V_{1}+V_{2}},
$$

where $c_{M_{i}}$ is the molar concentration of ions of $i$ th metal in the initial aqueous solution of corresponding salt, $\mathrm{mol} \mathrm{dm}^{-3} ; x_{i}$ is the number of metal atoms in the empirical formula of the salt of the corresponding $i$ th metal ( $x_{i}=1$ for the salts under study); and $v_{i}$ is the volume of aqueous solution of $i$ th salt added to basic RMS, $\mathrm{dm}^{3}$. The total water content in the RMS is given in the denominator.

During synthesis of bimetallic PdNi NPs, the metal ratio corresponded to $1: 1(\mathrm{~mol})$, and this value was $27: 1$ (mol) in the case of Fe/Pd NPs.

The amounts of metal ions per RMS volume were calculated as follows:

$$
c_{M_{i}}^{\mathrm{RMS}}=\frac{c_{M_{i}} x_{i} V_{i}}{V},
$$

where $V$ is the volume of reverse-micellar solution, $\mathrm{dm}^{3}$, and the other parameters are the same as in Eq. (2).

Molar concentrations of metal ions in RMS (3) $[\mathrm{Ni}]_{\mathrm{RMS}}$ and $[\mathrm{Pd}]_{\mathrm{RMS}}$ during preparation of monometallic NPs corresponded to $202 \mu \mathrm{mol} \mathrm{dm}^{-3}$; in the case of bimetallic PdNi NPs, $101 \mu \mathrm{mol} \mathrm{dm} \mathrm{dm}^{-3}$; and, in $\mathrm{Fe} / \mathrm{Pd} \mathrm{NPs}$, concentration of $[\mathrm{Fe}]_{\mathrm{RMS}}=4010 \mu \mathrm{mol}$ $\mathrm{dm}^{-3}$ and $[\mathrm{Pd}]_{\mathrm{RMS}}=150 \mu \mathrm{mol} \mathrm{\textrm {dm } ^ { - 3 }}$.

Bimetallic PdNi NPs were prepared through mutual reduction of metal ions in RMS upon simultaneous mixing of nickel and palladium salts $(1: 1$ $\mathrm{mol} / \mathrm{mol}$ ) with an expected internal structure of "alloy" type. $\mathrm{Fe} / \mathrm{Pd}$ nanoparticles with the core/shell structure $\mathrm{Fe}_{\text {core }} \mathrm{Pd}_{\text {shell }}$ were prepared through the addition of $10 \mu \mathrm{L}$ of aqueous solution of palladium salt with the concentration of $0.015 \mathrm{M}$ to $1 \mathrm{~mL}$ of RMS with stable (more than 25 months) Fe NPs $\omega=5.0$ prepared from the aqueous solution of iron salt with the concentration of $0.3 \mathrm{M}$. The intrinsic absorption spectrum of Fe NPs (Fig. 1) shows peaks at $\lambda \sim 240$, $\sim 274$, and $307 \mathrm{~nm}$.

The environmental temperature was $20-25^{\circ} \mathrm{C}$ during synthesis and instrumental measurements. The process was carried out in air in $25-\mathrm{cm}^{3}$ conical flasks with ground stoppers made from glass of TS brand; in the case of $\mathrm{Fe} / \mathrm{Pd} \mathrm{NPs}$, it was carried out in $1.5-\mathrm{mL}$ polypropylene microcentrifugal test tube of Eppendorf type.

\section{Preparation of Nanocomposites}

Metal NPs were deposited onto the surface of $\gamma-\mathrm{Al}_{2} \mathrm{O}_{3}$ carrier from RMS through impregnation. To prepare each nanocomposite, a total of $3 \mathrm{~mL}$ of each RMS per $1.00 \mathrm{~g} \gamma-\mathrm{Al}_{2} \mathrm{O}_{3}$ was taken; in the case of $\mathrm{Fe} / \mathrm{Pd} \mathrm{NPs}, 1 \mathrm{~mL}$ per $0.50 \mathrm{~g}$ of alumina. The time of adsorption of nanoparticles corresponded to $90 \mathrm{~min}$; in the case of $\mathrm{Fe} / \mathrm{Pd} \mathrm{NPs}, 5$ days.

To eliminate organic residues, after adsorption, the nanocomposite specimens were washed three times with $n$-heptane ( $1 \mathrm{~mL}$ each) and dried in the air over $24 \mathrm{~h}$. 


\section{Equipment}

The formation of nanoparticles during synthesis and their adsorption by the carrier were controlled by electronic absorption spectrophotometry using a Hitachi U-3310 instrument in the wavelength range of 190-800 $\mathrm{nm}$ and quartz cuvettes with the optical path length of $1 \mathrm{~mm}$. Scanning was performed from long to short waves. A solution of $0.15 \mathrm{~mol} \cdot \mathrm{dm}^{-3}$ AOT in isooctane was used as a reference specimen. Optical absorbance, rather than attenuation, was chosen as a criterion of the beam intensity transmitted through solution, because light scattering can be neglected for these specimens and the effect on the form of the spectrum caused by fluorescence of the specimen was eliminated by scanning the spectrum from long to short waves.

Synthesis of NPs was also controlled by fluorescence spectroscopy using a Hitachi F-7000 instrument. Emission spectra were recorded in the scanning mode of wavelengths upon excitation of the specimen by electromagnetic radiation at the wavelength of $255 \mathrm{~nm}$. Scanning was performed in the range from 250 to $600 \mathrm{~nm}$ at a scan rate of $1200 \mathrm{~nm} / \mathrm{min}$ and a data acquisition step of $1 \mathrm{~nm}$. The spectral slit width values corresponded to $5 \mathrm{~nm}$ for excitation and emission fluxes. The emission spectrum was recorded at an angle of $90^{\circ}$ to the excitation beam. The voltage on the photoelectronic multiplier corresponded to $700 \mathrm{~V}$, and the response constant was $0.5 \mathrm{~s}$. The spectra were recorded without correction on the linear characteristics of the source of excitation and detection system. To eliminate Rayleigh scattering in the recorded spectra, as well as its second harmonics, a UV-29 filter was mounted along the line of the excitation beam with cut-off of the ultraviolet range of $\sim 290 \mathrm{~nm}$. The specimen was transferred to a quartz cuvette made from synthetic (nonfluorescent) quartz with an internal width of $10 \mathrm{~mm}$.

NP sizes were determined using atomic force microscopy (AFM) on a MultiMode instrument (Bruker) equipped with NSG-01 silicon cantilevers (NT-MDT) in the tapping mode. The resolution by height was \pm 0.1 and $\pm 12 \mathrm{~nm}$ along a lateral line. Solutions of NPs were deposited onto an atomically smooth layer of mica substrate. After that, the substrate was dried in air, washed with water, and dried again. The specimens were left in the room with the atomic force microscope before measurements to equilibrate their temperatures, which eliminated distortion of the results caused by temperature drift. The images were processed in the WSxM 5.0 program $[4,7]$. The height of the particles was taken as their size (the $z$ parameter).

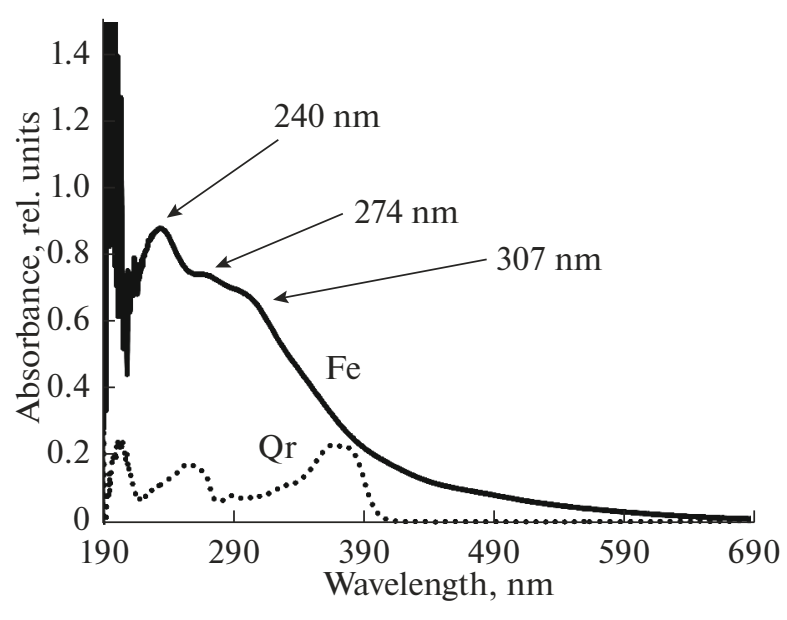

Fig. 1. Absorption spectra of RMS $\omega=5.0$ with initial Fe NPs. Qr is the spectrum of the Qr/AOT/isooctane system.

\section{RESULTS AND DISCUSSION \\ Synthesis of Metal Nanoparticles. Electronic Spectrophotometry}

Figure 2 shows the spectra of (a) Pd, (b) Ni, (c) $\mathrm{PdNi}$, and (d) Fe/Pd NPs with the initial spectrum of quercetin (Qr). The formation of palladium nanoparticles is characterized by a decrease in the absorption intensity at $218 \mathrm{~nm}$ over the first $60 \mathrm{~min}$ followed by an increase over the subsequent 7 days; during the 67th day of the synthesis, changes of the spectrum could be observed, in particular, in the long-wavelength range, which indicates long-term changes in the chosen system. Pd nanoparticles (Fig. 2a) are characterized by peaks with maxima at $\lambda \sim 220, \sim 272$, and $\sim 327 \mathrm{~nm}$, which agrees with the previous data for Pd NPs synthesized through the radiation-chemical method [810], as well as theoretical calculations and data obtained by other methods [11-13]. Analogously with palladium NPs, Ni NPs (Fig. 2b) are characterized by absorption in the UV region of 210-220 nm [14], which is caused by surface plasmon resonance; however, it possesses a slightly broader band, which is related to the presence of the second neighboring band, though a less intense one. It should be noted that Ni NPs are formed in a different way than are palladium NPs; this is indicated by the residual quercetin content in RMS with metal NPs after 7 days of synthesis with intrinsic peaks in the range of 365-390 nm corresponding to absorption of the B-ring of the cinnamoyl system of quercetin. However, after 67 days, there is a nearly double decrease in the absorption intensity of quercetin, whereas the absorption intensity of the NPs increases by $40 \%$.

The spectrum of the RMS of bimetallic PdNi NPs (Fig. 2c) with the expected structure of a homogeneous alloy is characterized by the superposition of individual absorption bands of plasmon resonances of nickel and palladium, which is larger than the alge- 
(a)

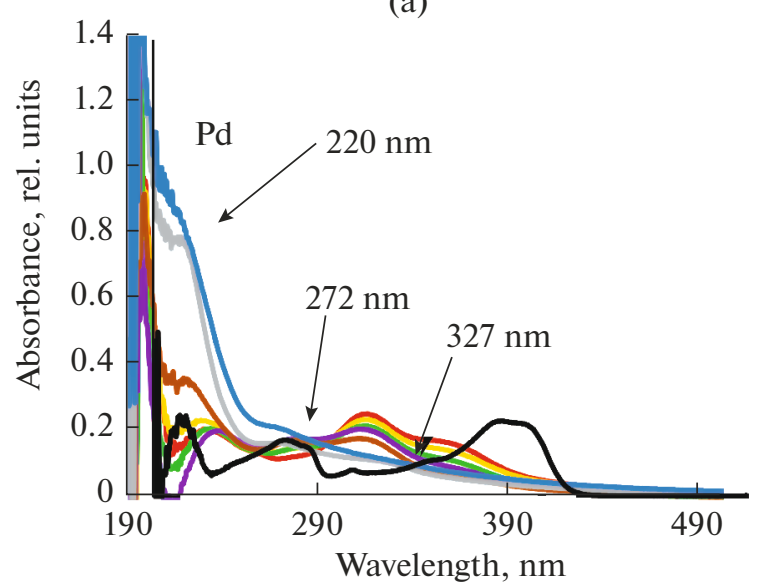

(c)

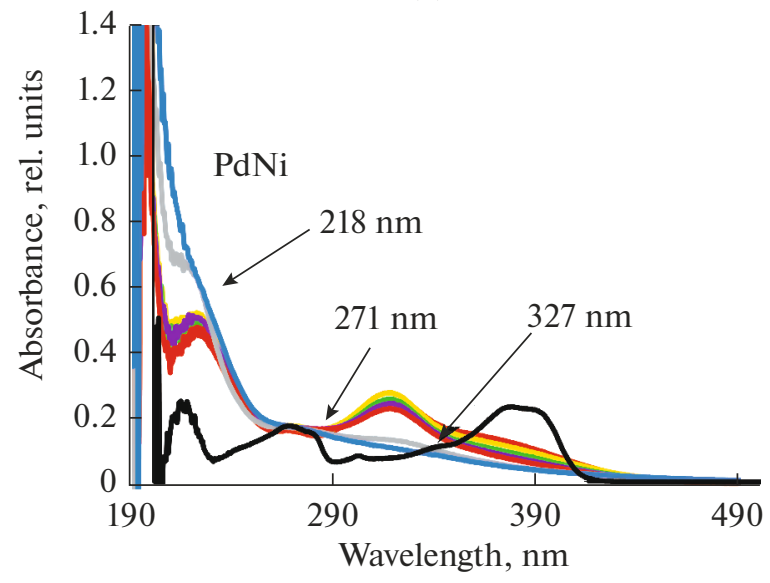

(b)

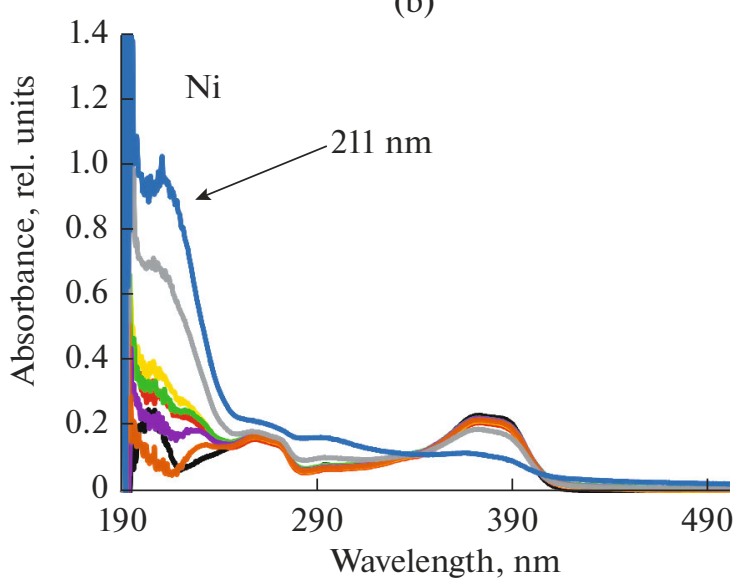

(d)

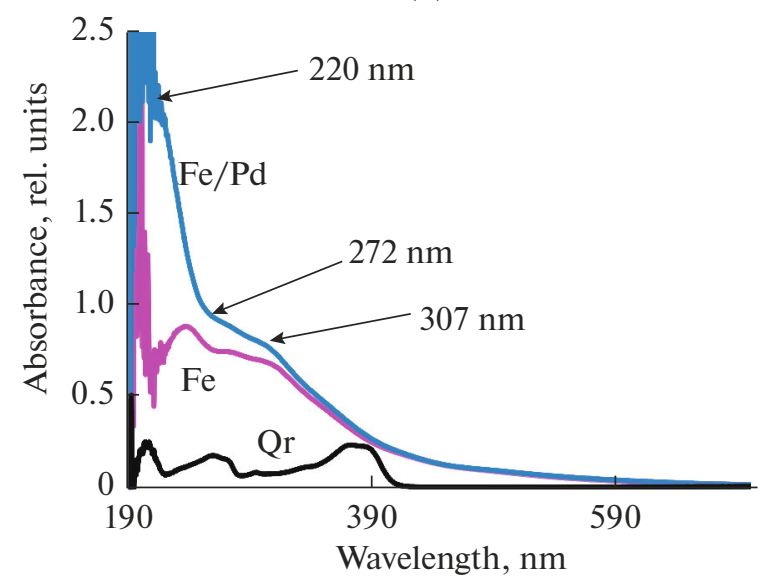

Fig. 2. Absorption spectra of RMS $w=5.0$ with (a) Pd NPs, (b) Ni NPs, (c) PdNi NPs, and (d) initial Fe NPs and formed Fe/Pd NPs (2 days): — $3 \mathrm{~min}, \_15 \mathrm{~min}, \_30 \mathrm{~min}, \_60 \mathrm{~min}, \_90 \mathrm{~min}, \_7$ days, and — — _ 67 days of synthesis. Qr (-) is the spectrum of Qr/AOT/isooctane solution.

braic sum of the spectra of each metal. Thus, there is a synergism in the spectral characteristics of formed PdNi NPs, which is confirmed by the formation of a bimetallic structure, which is finished after 7 days of the synthesis. In contrast to monometallic NPs, there is no decrease in the absorption intensity in the deepultraviolet range during this synthesis. A maximum of $\lambda$ at $\sim 218 \mathrm{~nm}$ was recorded in the spectrum, which is comparable to the absorption maximum of Pd NPs and broader due to the absorption of Ni NPs. The latter two peaks are caused by the presence of palladium atoms in nanoparticles, whereas the former possesses a maximum that is comparable to the maximum of monometallic Pd NPs and broader than that of Ni NPs.

During synthesis, reverse-micellar solutions change color from transparent colorless to light-yellow.

Iron nanoparticles (Fig. 1) possess intrinsic peaks in UV-region with the maxima at 240, 274, and $307 \mathrm{~nm}$, which agrees with the literature data [6]. After addition of palladium and the formation of bimetallic $\mathrm{Fe} / \mathrm{Pd}$
NPs, there is a significant increase in the absorbance intensity at $\lambda \sim 220 \mathrm{~nm}$ and less remarkable in the range from 250 to $320 \mathrm{~nm}$ (Fig. 2d). On the basis of these data, the formation of the layer from finer Pd NPs on the surface of Fe NPs can be suggested.

\section{Fluorescence Spectroscopy}

Fluorescence spectrum of micellar solution of AOT/isooctane (Fig. 3a, AOT) is characterized by the peaks at 314 and $453 \mathrm{~nm}$. With the addition of quercetin (Fig. 3a, Qr) to this solution, the fluorescence intensity of the latter decreases nonuniformly in various ranges resulting in the shift of the peak maxima. This fact can be rationalized by the acceptor binding of impurity metal ions by quercetin. The shift of the absorption bands is experimentally recorded due to the formed complexes with the bond of Me ions with various - $\mathrm{OH}$ groups of Qr. Emission peaks of quercetin at 549 and $416 \mathrm{~nm}$ also arise. 
After the addition of nickel ions to the Qr/AOT/isooctane system (Fig. 3a, Ni), the fluorescence peak intensity of Qr $(549 \mathrm{~nm})$ decreases by a factor of 3.8. Quercetin only partially binds nickel ions under these conditions (nickel ions decrease the intensity of quercetin during the formation of the complex $\mathrm{Qr}: \mathrm{Ni}=1: 2$ under saturation conditions [15]); however, the complexes at a $1: 1$ ratio are formed at lower concentration of nickel ions [16]. The presence of quercetin peak after 67 days of synthesis indicates a slow rate of reduction of nickel ions under these conditions. The peak with the highest intensity at $338 \mathrm{~nm}$ can be attributed both to primary intermediate products of quercetin oxidation and their nickel complexes.

It should be noted that the addition of palladium ions to the Qr/AOT/isooctane system (Fig. 3b, Pd) results in a total disappearance of quercetin peak at the first day of synthesis. Palladium should quench quercetin emission to a higher extent than nickel. First, the complexes at a $1: 1$ molar ratio are formed, which are slightly more stable than nickel complexes [16]. Enhancement of the peak at $\lambda \sim 450 \mathrm{~nm}$ was noted, which can be attributed to the complex of palladium ions with quercetin and molecular oxygen.

During mutual addition of nickel and palladium ions (with concentrations being twice as low as in previous cases) (Fig. 3c, PdNi), the form of the spectrum is mainly similar to the form of the spectrum for palladium, which indicates that a predominant role is played by palladium ions in the complex formation and, presumably, their reduction and NP formation. The rate of disappearance of free quercetin in this case is maximum. Such change of the spectra can indicate the formation of core/shell nanoparticles, with Pd NPs as core and nickel as shell.

\section{Sizes of Nanoparticles}

The sizes of metal nanoparticles were determined after their formation and maintenance over 7 days before their adsorption onto the carrier. The results are given in Table 2. Figure 4 shows an example topographic image of the mica substrate with the deposited Pd NPs and their histogram with size distribution. A sufficiently narrow NP size distribution should be noted, which is characteristic for this method of synthesis at low solubilization coefficient. Ni NPs possess similar characteristics, while bimetallic PdNi NPs are represented by the aggregates from finer nanoparticles (only aggregate sizes are given for them in Table 2, because the sizes of individual NPs could not be determined). Table 2 shows the estimated data on the mean number of atoms in monometallic nanoparticle assuming that all ions are reduced and the particles are spherical, which was calculated using the following equation [17]:

$$
N_{\mathrm{at}}=\frac{V_{\mathrm{NP}}}{V_{\mathrm{a}}}=\left(\frac{d_{\mathrm{NP}} / 2}{r_{\mathrm{WS}}}\right)^{3},
$$
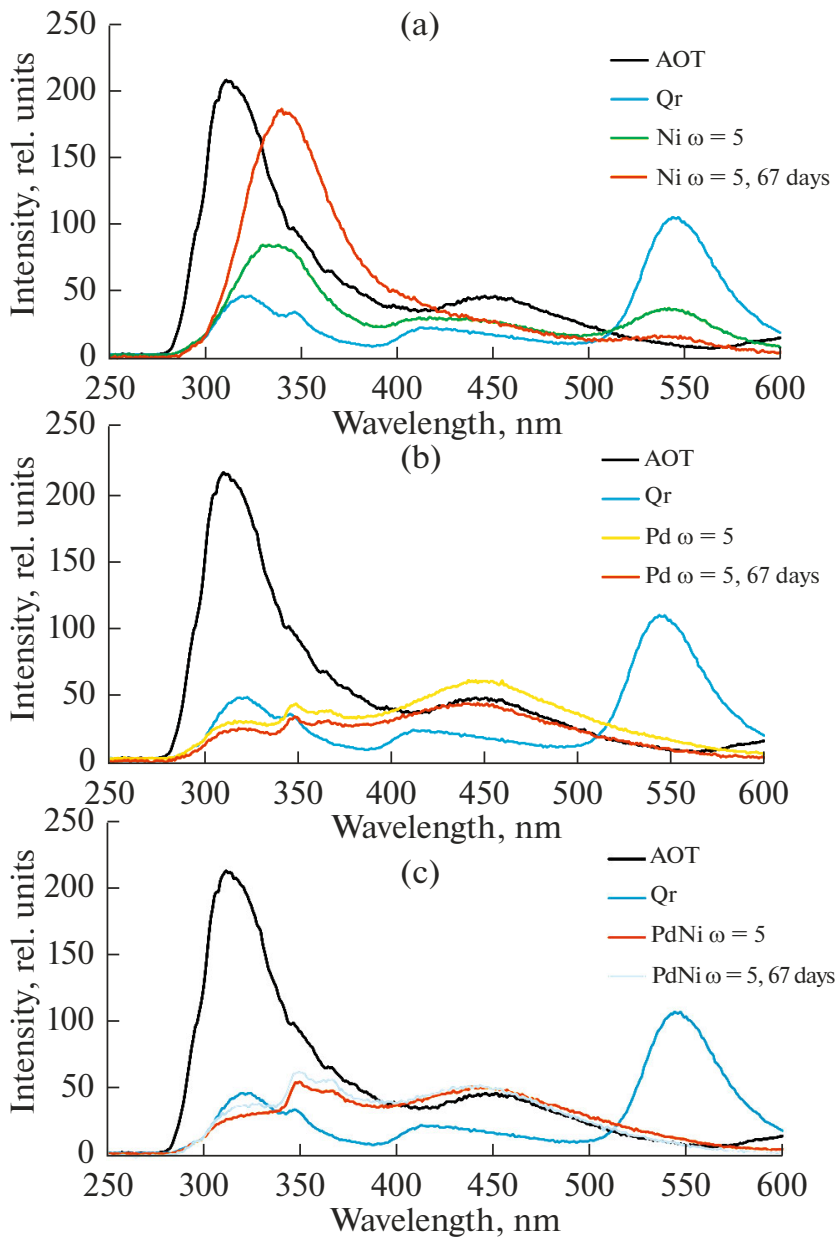

Fig. 3. Fluorescence spectra of RMS solutions with (a) $\mathrm{Ni}$ NPs, (b) Pd NPs, and (c) PdNi NPs at the day of synthesis and after 67 days.

where $d_{\mathrm{NP}}$ is the mean diameter of the NPs determined by AFM, nm, and $r_{\mathrm{WS}}$ (is the radius of WignerSeitz cell, $\mathrm{nm}$. In the case of $\mathrm{Pd}, r_{\mathrm{wS}}=0.152 \mathrm{~nm}$; and $\mathrm{Ni}, 0.138 \mathrm{~nm}$ [18]. $N_{\text {at }}$ is the mean number of metal atoms in one nanoparticle.

The mean concentration of NPs in solution, which is convenient for evaluation of the number of catalytic centers of nanocomposite, was calculated using the following equation:

$$
c_{\mathrm{NP}}(M e)=\frac{c_{M}^{\mathrm{RMS}}(M e) N_{\mathrm{A}}}{N_{\mathrm{at}}(M e)},
$$

where $c_{M}^{\mathrm{RMS}}(\mathrm{Me})$ is the number of Me metal ions per volume of RMS, mol dm ${ }^{-3} ; N_{\text {at }}(M e)$ is the mean number of atoms in one NP calculated from AFM data; and $N_{\mathrm{A}}$ is the Avogadro number. 
Table 2. Parameters of NPs

\begin{tabular}{l|c|c|c}
\hline \multicolumn{1}{c|}{ RMS } & Mean diameter, $\mathrm{nm}$ & Number of atoms in NP & Concentration of NPs, NP $\mathrm{dm}^{-3}$ \\
\hline Ni $\omega=5.0$ & 1.1 & 63 & $1.8 \times 10^{18}$ \\
Pd $\omega=5.0$ & 1.3 & 78 & $1.5 \times 10^{18}$ \\
PdNi $\omega=5.0$ & 4.4 & - & - \\
\hline
\end{tabular}

\section{Preparation of Nanocomposites}

During preparation of the nanocomposites based on $\gamma-\mathrm{Al}_{2} \mathrm{O}_{3}$, a nearly double decrease in the absorption intensity in the range of the localized plasmon resonance of Pd and Ni metal and PdNi bimetal NPs was recorded according to the results of spectrophotometric control of adsorption of NPs from reverse-micellar solution (Fig. 5). Optical absorbance of $\mathrm{Fe} / \mathrm{Pd} \mathrm{NP}$ in RMS after the end of adsorption on $\gamma-\mathrm{Al}_{2} \mathrm{O}_{3}$ decreases to zero values in the visible wavelength range starting from $\lambda \sim 250 \mathrm{~nm}$. A drastic change in the absorbance intensity of this specimen in the range from 190 to $250 \mathrm{~nm}$ and its decrease below zero value can be related to the adsorption of not only Fe/Pd NPs, but also AOT by the carrier, the absorption band of which is also in the same range of the spectrum [8]. In this case, the absorption intensity of AOT in the $0.15 \mathrm{M}$ system of AOT/isooctane composition is lower than the absorbance of $\mathrm{Fe} / \mathrm{Pd}$ NPs in RMS in this wavelength range, which allows us to state that there has been full adsorption of $\mathrm{Fe} / \mathrm{Pd}$ NPs recorded in the UV region of the spectrum. During adsorption of $\mathrm{Ni}$ and $\mathrm{PdNi}$, there is an increase in the absorption intensity at $\Delta \mathrm{t}=60 \mathrm{~min}$ for $\mathrm{Ni}$ and $\Delta t=10 \mathrm{~min}$ for $\mathrm{PdNi}$, as well as more rapid termination of this process as compared to $\mathrm{Pd}$.

The theoretical content of the metal in nanocomposites is less than $36 \mu \mathrm{g} / \mathrm{g}\left(3.9 \times 10^{15} \mathrm{NP} / \mathrm{g}\right)$ for $\mathrm{Ni}$ /
$\gamma-\mathrm{Al}_{2} \mathrm{O}_{3} ; 65 \mu \mathrm{g} / \mathrm{g}\left(3.6 \times 10^{15} \mathrm{NP} / \mathrm{g}\right)$ in $\mathrm{Pd} / \gamma-\mathrm{Al}_{2} \mathrm{O}_{3}$; $32 \mu \mathrm{g} / \mathrm{g}$ and $18 \mu \mathrm{g} / \mathrm{g}$ of palladium and nickel, respectively, in $\mathrm{PdNi} / \gamma-\mathrm{Al}_{2} \mathrm{O}_{3}$; and $32 \mu \mathrm{g} / \mathrm{g}$ palladium and $452 \mu \mathrm{g} / \mathrm{g}$ of iron in $\mathrm{Fe} / \mathrm{Pd} / \gamma-\mathrm{Al}_{2} \mathrm{O}_{3}$. Effectiveness of adsorption and maximum possible theoretical content of metal NPs in nanocomposites can be evaluated from the data in Table 3. Effectiveness of sufficiently large Fe NPs as transport particles for palladium NPs formed on their surface should be noted. Concentrations of palladium in initial solutions of monometallic and bimetallic NPs are comparable, 21.55 and $12.80 \mathrm{mg} \mathrm{dm}^{-3}$, respectively, whereas the adsorption effectiveness (according to the fraction of adsorbed particles) in the case of bimetallic Fe/Pd NPs is twice as large.

Qualitative determination of the retention of metal NPs on the substrate surface and simultaneous removal of residual organic compounds were performed through washing of the nanocomposites with $n$-heptane. Figure 6 shows example spectra of three subsequent washes of Ni and Pd NPs from the adsorbent surface. A sufficiently strong retention of nickel nanoparticles on the surface of $\gamma-\mathrm{Al}_{2} \mathrm{O}_{3}$ and weak retention of palladium particles on it were mentioned, which affects residual content of Pd NPs in the ready catalyst. The nanocomposite with the composition $\mathrm{PdNi} / \gamma-\mathrm{Al}_{2} \mathrm{O}_{3}$ (Fig. 7) displays similar characteristics of retention of NPs with the $\mathrm{Ni} / \gamma-\mathrm{Al}_{2} \mathrm{O}_{3}$ composite
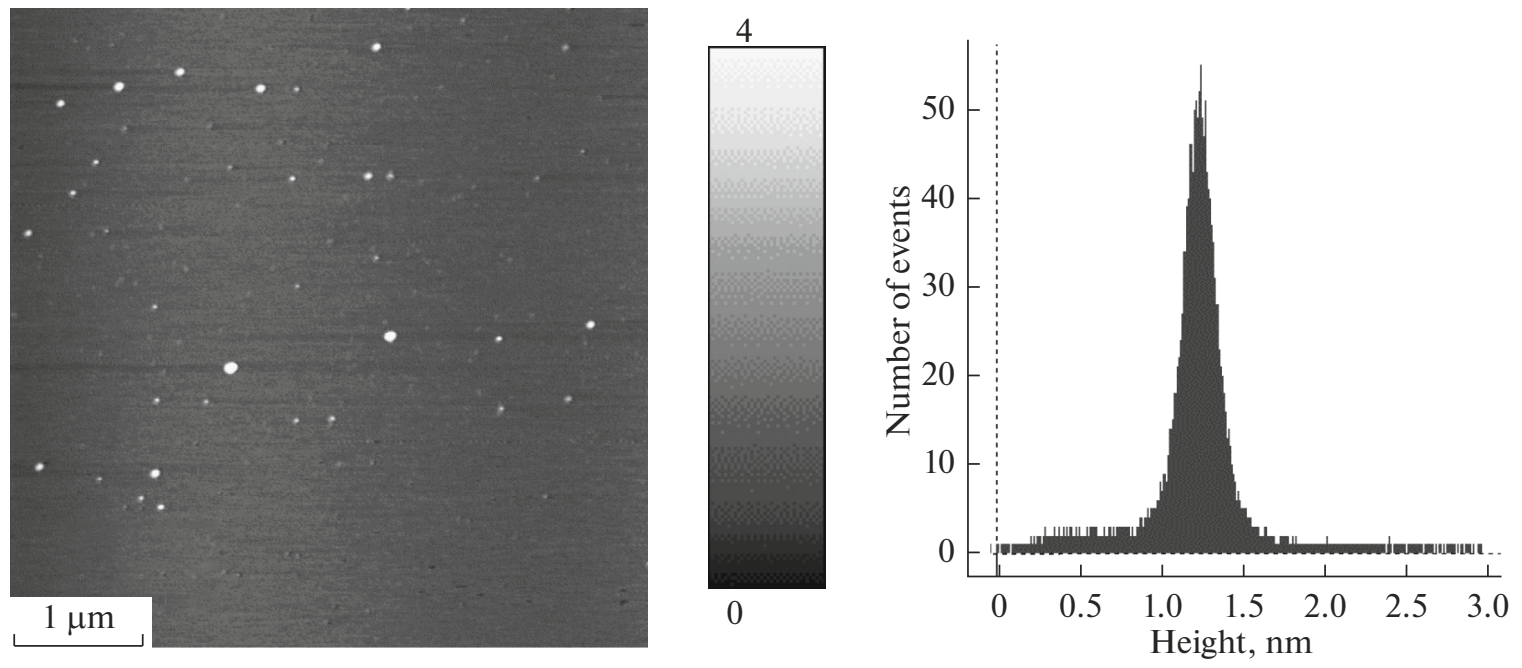

Fig. 4. AFM image and the histogram of particle size distribution of Pd NPs. 

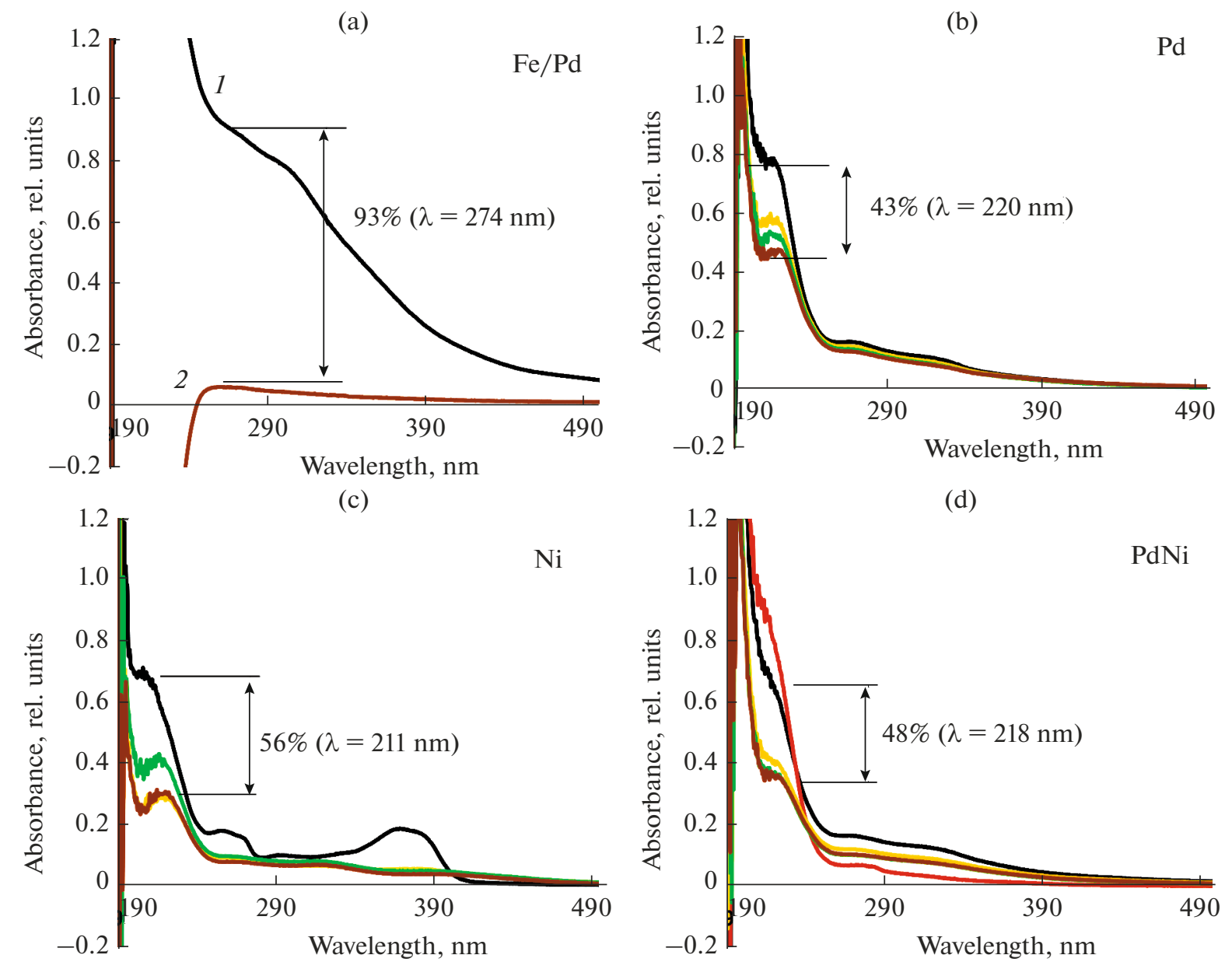

Fig. 5. Change of the spectra of RMS during adsorption: — initial, _ $10 \mathrm{~min}, \longleftarrow 30 \mathrm{~min}, \longleftarrow 60 \mathrm{~min}$, and — $-90 \mathrm{~min}$. Percentage of the decrease in the absorption intensity after adsorption is given.

differing by a slightly reduced degree of retention, whereas highest retention of NPs among all considered composites is characterized in the case of $\mathrm{Fe} / \mathrm{Pd} / \gamma-\mathrm{Al}_{2} \mathrm{O}_{3}$ specimen (Fig. 7).

\section{CONCLUSIONS}

The formation of bimetallic structure of PdNi NPs has been confirmed by a synergetic character of enhancement of optical absorbance of their solution as compared to the absorbance of the solutions of individual monometallic NPs. Analysis of fluorescence spectra indicates possible formation of the core/shell structure, in which Pd is core. The use of large particles has increased the adsorption effectiveness of $\mathrm{Pd}$ $\mathrm{NPs}$ in the form of $\mathrm{Fe} / \mathrm{Pd}$ bimetal by the $\gamma$-alumina surface due to stronger binding. This has increased the degree of recovery of metals from solutions [19] by the surface of carrier, which decreases the amount of expensive metal and reduces the costs for the produc-

Table 3. Calculated metal content in the nanocomposites

\begin{tabular}{l|c|c|c|c|c|c|c}
\hline \multirow{2}{*}{\multicolumn{1}{c}{ Specimen }} & \multirow{2}{*}{$\alpha, \%$} & \multicolumn{3}{|c|}{ Metal content in nanocomposite, $\mu \mathrm{g} / \mathrm{g}(\mathrm{ppm})$} & \multicolumn{3}{c}{ Metal content in nanocomposite, $\mu \mathrm{mol} / \mathrm{g}$} \\
\cline { 3 - 8 } & & $\mathrm{Pd}$ & $\mathrm{Fe}$ & $\mathrm{Ni}$ & $\mathrm{Pd}$ & $\mathrm{Fe}$ & $\mathrm{Ni}$ \\
\hline Pd NP in RMS & 43 & 28 & - & - & 0.26 & - & - \\
Ni NP in RMS & 56 & - & - & 20 & - & - & 0.34 \\
PdNi NP in RMS & 48 & 15 & - & 9 & 0.14 & - & 0.15 \\
Fe/Pd NP in RMS & 93 & 30 & 420 & - & 0.28 & 7.53 & - \\
\hline
\end{tabular}

$\alpha$ is the fraction of adsorbed NPs (corresponds to the percentage of the decrease in the absorbance intensity after adsorption). 
(a)

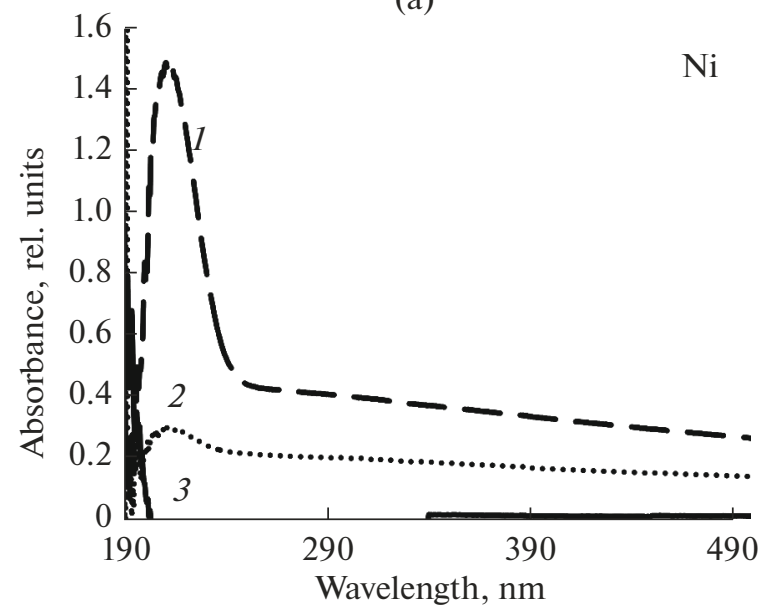

(b)

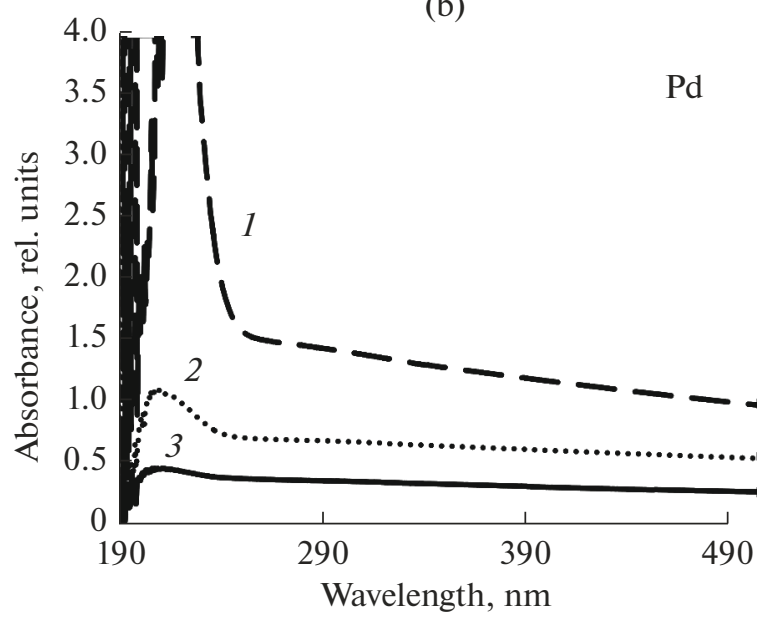

Fig. 6. Spectra of the washes from nanocomposites based on Ni and Pd NPs. (1) First wash, (2) second wash, and (3) third wash.

(a)

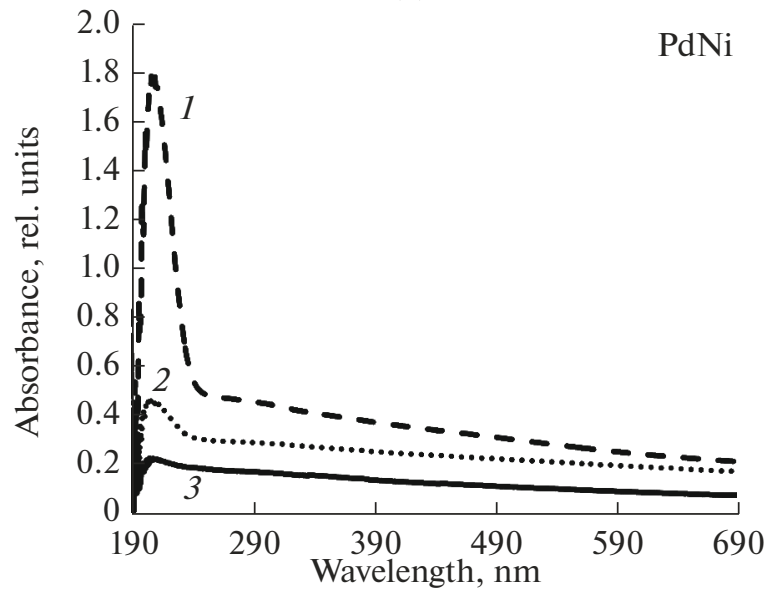

(b)

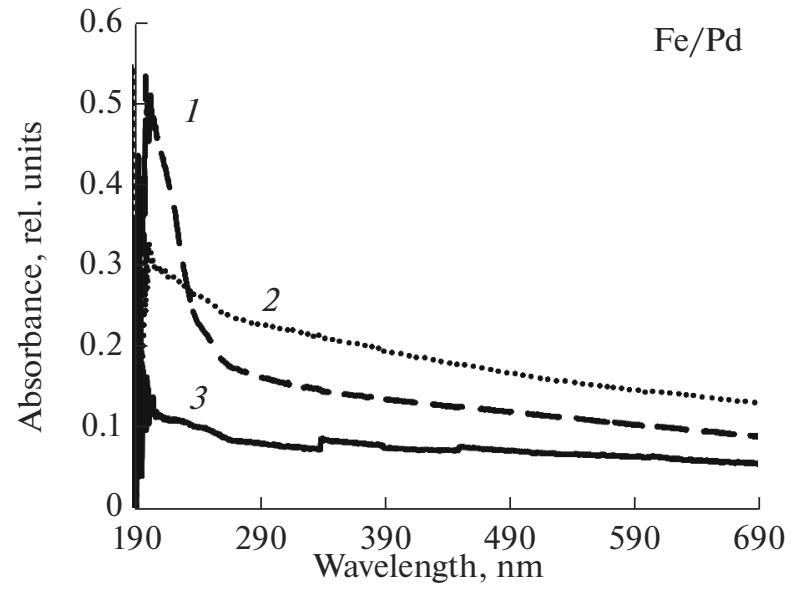

Fig. 7. Spectra of the washes from nanocomposite based on PdNi and Fe/Pd NPs. (1) First wash, (2) second wash, and (3) third wash.

tion of nanocomposite materials based on the considered transition metals.

\section{ACKNOWLEDGMENTS}

The sizes of metal nanoparticles were measured using the equipment of the Center of Collective Use of Physical Methods of Study of the Frumkin Institute of Physical Chemistry and Electrochemistry of the Russian Academy of Sciences.

\section{OPEN ACCESS}

This article is licensed under a Creative Commons Attribution 4.0 International License, which permits use, sharing, adaptation, distribution and reproduction in any medium or format, as long as you give appropriate credit to the original author(s) and the source, provide a link to the Creative Commons licence, and indicate if changes were made. The images or other third party material in this article are included in the article's Creative Commons licence, unless indicated otherwise in a credit line to the material. If material is not included in the article's Creative Commons licence and your intended use is not permitted by statutory regulation or exceeds the permitted use, you will need to obtain permission directly from the copyright holder. To view a copy of this licence, visit http://creativecommons.org/licenses/by/4.0/.

\section{REFERENCES}

1. Walther, A. and Müller, A.H.E., Chem. Rev., 2013, vol. 113, no. 7, pp. 5194-5261.

https://doi.org/10.1021/cr300089t 
2. Gilroy, K.D., Ruditskiy, A., Peng, H.-C., et al., Chem. Rev., 2016, vol. 116, no. 18, pp. 10414-10472. https://doi.org/10.1021/acs.chemrev.6b00211

3. Sergeev, M.O., Revina, A.A., Boeva, O.A., Zhavoronkova, K.N., and Zolotarevskii, V.I., Prot. Met. Phys. Chem. Surf., 2020, vol. 56, no. 1, pp. 63-74. https://doi.org/10.1134/S2070205120010207

4. Chagas, C.A., Manfro, R.L., and Toniolo, F.S., Catal. Lett., 2020, vol. 150, pp. 3424-3436. https://doi.org/10.1007/s10562-020-03257-1

5. Revina, A.A., RF Patent 2312741, 2007.

6. Revina, A.A., Suvorova, O.V., Pavlov, Yu.S., and Tytik, D.L., Prot. Met. Phys. Chem. Surf., 2019, vol. 55, no. 5 , pp. $888-894$. https://doi.org/10.1134/S2070205119040166

7. Horcas, I., Fernandez, R., Gomez-Rodriguez, J.M., et al., Rev. Sci. Instrum., 2007, vol. 78, p. 013705. https://doi.org/10.1063/1.2432410

8. Revina, A.A., RF Patent 2322327, 2008.

9. Revina, A.A., Belyakova, L.D., Parkaeva, S.A., Suvorova, O.V., Sergeev, M.O., and Zolotarevski, V.I., Prot. Met. Phys. Chem. Surf., 2010, vol. 46, no. 6, pp. 648654. https://doi.org/10.1134/S2070205110060043

10. Sergeev, M.O., Revina, A.A., Busev, S.A., Zolotarevskiy, V.I., Zhavoronkova, K.N., and Boeva, O.A., Nanotechnol. Rev., 2014, vol. 3, no. 5, pp. 515-525. https://doi.org/10.1515/ntrev-2014-0011

11. Creighton, A.J. and Eadon, D.G., J. Chem. Soc., Faraday Trans., 1991, vol. 87, no. 24, pp. 3881-3891. https://doi.org/10.1039/FT9918703881
12. Teranishi, T. and Miyake, M., Chem. Mater., 1998, vol. 10, pp. 594-600.

https://doi.org/10.1021/cm9705808

13. Shaochun Tang, Sascha Vongehr, Zhou Zheng, Hua Ren, and Xiangkang Meng, Nanotechnology, 2012, vol. 23 , p. 255606. https://doi.org/10.1088/0957-4484/23/25/255606

14. Revina, A.A., Magomedbekov, E.P., and Veretennikova, G.V., Radiochemistry, 2018, vol. 60, no. 1, pp. 6469.

15. Kakavand, K., Niknam, V., Faridbod, F., Ebrahimzadeh, H., and Hamidipour, A., Anal. Bioanal. Chem., 2012, vol. 4, no. 6, pp. 635-645.

16. Malesev, D. and Kuntic, V., J. Serb. Chem. Soc., 2007, vol. 72 , no. 10 , pp. $921-939$. https://doi.org/10.2298/JSC0710921M

17. Mazalova, V.L., Kravtsova, A.N., and Soldatov, A.V., Nanoklastery: rentgenospektral'nye issledovaniya $i$ komp'yuternoe modelirovanie (Nanoclusters: X-Ray Spectral Researches and Computer Simulation), Moscow: Fizmatlit, 2013.

18. Ren, F., Cao, K., Ren, J., et al., J. Comput. Theor. Nanosci., 2014, vol. 11, no. 2, pp. 344-347. https://doi.org/10.1166/jctn.2014.3358

19. Tsivadze, A.Yu., Herald Russ. Acad. Sci., 2020, vol. 90, no. 2, pp. 214-224. https://doi.org/10.1134/S1019331620020185

Translated by A. Muravev 